\title{
Interpreting Eddy Fluxes
}

\author{
CARSTEN EDEN \\ Leibniz-Institut für Meereswissenschaften, IFM-GEOMAR, Kiel, Germany \\ RichaRd J. GREATBATCH \\ Department of Oceanography, Dalhousie University, Halifax, Nova Scotia, Canada \\ Dirk Olbers \\ Alfred Wegener Institute for Polar and Marine Research, Bremerhaven, Germany
}

(Manuscript received 25 May 2005, in final form 9 August 2006)

\begin{abstract}
A generalization of the transformed Eulerian and temporal residual means is presented. The new formulation uses rotational fluxes of buoyancy, and the full hierarchy of statistical density moments, to reduce the cross-isopycnal eddy flux to the physically relevant component associated with the averaged water mass properties. The resulting eddy-induced diapycnal diffusivity vanishes for adiabatic, statistically steady flow, and is related to either the growth or decay of mesoscale density variance and/or the covariance between small-scale forcing (mixing) and density fluctuations, such as that associated with the irreversible removal of density variance by dissipation. The relationship between the new formulation and previous approaches is described and is illustrated using results from an eddying channel model. The formalism is quite general and applies to all kinds of averaging and to any tracer (not just density).
\end{abstract}

\section{Introduction}

The Boussinesq form of the conservation equation for a tracer with concentration $b$ in the ocean (or the atmosphere) is given by

$$
\frac{\partial b}{\partial t}+\nabla \cdot(\mathbf{u} b)=Q
$$

where $\mathbf{u}$ denotes the instantaneous, three-dimensional velocity and $Q$ is a forcing term. Both the ocean and atmosphere are turbulent fluids, full of "rapidly evolving perturbations" (eddies) on a "slowly evolving mean state." The presence of the eddies means that the instantaneous tracer distribution is often of little interest; instead, it is the dynamics and evolution of an "averaged" state that is important. This, in turn, requires the definition of an average or filter with which to view the dynamics and evolution of the tracer field, and which,

Corresponding author address: Dr. Carsten Eden, IFMGEOMAR, FB I, Ocean Circulation and Climate Dynamics, Düsternbrooker Weg 20, 24105 Kiel, Germany.

E-mail: ceden@ifm-geomar.de in turn, determines what is meant by "rapid," "slow," and "mean state."

A simple example is the zonal mean; that is, $b, \mathbf{u}$, and $Q$ are decomposed into zonal averages at a constant height and deviations from that average,

$$
\bar{b}=\left(x_{2}-x_{1}\right)^{-1} \int_{x_{1}}^{x_{2}} b d x \quad \text { and } \quad b^{\prime}=b-\bar{b},
$$

and correspondingly for $\mathbf{u}$ and $Q$. Substituting the decomposition given by Eq. (2) into the instantaneous tracer budget and averaging the result leads to the equation for the mean tracer $\bar{b}$ given by

$$
\frac{\partial \bar{b}}{\partial t}+\boldsymbol{\nabla} \cdot(\overline{\mathbf{u}} \bar{b})+\boldsymbol{\nabla} \cdot\left(\overline{\mathbf{u}^{\prime} b^{\prime}}\right)=\bar{Q} .
$$

An immediate difficulty is presented by the eddy tracer flux ${ }^{1} \overline{\mathbf{u}^{\prime} b^{\prime}}$. These fluxes couple the mean tracer budget to that of the perturbations, such that the evolution of

\footnotetext{
${ }^{1}$ Note that the vectors $\overline{\mathbf{u}}, \mathbf{u}^{\prime}$, the operator $\boldsymbol{\nabla}$, and correspondingly the fluxes $\overline{\mathbf{u}} \bar{b}$ and $\overline{\mathbf{u}^{\prime} b^{\prime}}$ in Eq. (2) and in the following section, are two-dimensional, that is, their zonal component vanishes.
}

DOI: $10.1175 / J P O 3050.1$ 
the perturbations has to be known to predict the mean tracer. Of course, the solution to this problem is thought to be given by parameterizing the perturbation quantities in terms of the mean quantities. However, before parameterizing the effect of the eddy tracer fluxes, it is necessary to understand and interpret them. Understanding and interpreting the eddy fluxes is the focus of the present paper.

Some insight into the nature of the eddy tracer flux can be obtained by considering a layered framework, in which instantaneous contours of $b$ are taken as layer interfaces. In the continuous limit of infinitesimal layer thickness, this is the same as using $b$ as the vertical coordinate. ${ }^{2}$ Taking $b$ to be potential density ${ }^{3}$ then corresponds to using "isopycnal coordinates" (e.g., McDougall 1987). Because the interior oceanic flow is almost adiabatic (Wüst 1935), the diabatic forcing $Q$ is expected to be small in the ocean interior and associated with weak diapycnal mixing. In the limit of vanishing instantaneous forcing $Q$, there is no instantaneous exchange of $b$ across layers (isopycnals) and it is easy to see that in the isopycnally averaged budget for $b$ there can be no cross-isopycnal flux. Furthermore, the mean forcing (related to $Q$ ) is controlling the crossisopycnal flux in the mean budget of the layer thickness.

Returning to $z$ coordinates, this property of the eddy fluxes is not as obvious. In fact, eddy tracer fluxes averaged at a constant height (instead of at constant $b$ ) usually show strong cross-isopycnal components even for the steady, weakly diabatic case, suggesting strong, apparently eddy-induced, diapycnal processes as we shall show below. On the other hand, $z$ coordinates are convenient and simple to use for both analytical considerations and numerical calculations. In fact, an overwhelming number of analytical and numerical models are based on $z$ coordinates, rather than either layered or isopycnal coordinates.

Therefore, it would be desirable if the character of eddy tracer fluxes as revealed above in the layered framework could be carried over to the mean tracer budget in $z$ coordinates. Based on these considerations, we can formulate the following statement, which we would like to apply in quasi-steady ${ }^{4}$ conditions in $z$ coordinates:

\footnotetext{
${ }^{2}$ This is the case, assuming that $b$ is a monotonic function of depth. See Nakamura (2001) and Nurser and Lee (2004) for a generalization of this approach for nonmonotonic functional forms of $b$.

${ }^{3}$ Note that we assume for simplicity an equation of state in which both potential density and neutral density are the same.

${ }^{4}$ We mean by quasi-steady state that $(\partial \bar{b} / \partial t)=0$ but, in general, $\left(\partial b^{\prime} / \partial t\right) \neq 0$.
}

(i) If there is no diabatic forcing $Q$ locally in the instantaneous tracer budget [Eq. (1)], there should be also no diabatic effects locally in the mean budget [Eq. (3)]. As a consequence, the divergence of the eddy tracer flux $\overline{\mathbf{u}^{\prime} b^{\prime}}$ must be entirely expressible as a divergence of an advective flux of the mean tracer.

For nonzero $Q$, on the other hand, we expect there to be diabatic effects resulting from the eddies that are contained in the divergence of $\overline{\mathbf{u}^{\prime} b^{\prime}}$ in the budget for $\bar{b}$. The diabatic nature of the eddy fluxes can be understood in an integral sense in $z$ coordinates as outlined by Radko and Marshall (2004).

Consider an integral over an area $A$ (or volume for the three-dimensional case and temporal averaging) above a mean isopycnal $\bar{b}(y, z)=$ const of the mean tracer budget [Eq. (3)] in, for instance, a cross section of a channel,

$$
\int_{A} d A\left[\boldsymbol{\nabla} \cdot(\overline{\mathbf{u}} \bar{b})+\boldsymbol{\nabla} \cdot\left(\overline{\mathbf{u}^{\prime} b^{\prime}}\right)\right]=\int_{A} d A\left(\bar{Q}-\frac{\partial}{\partial t} \bar{b}\right) .
$$

Using Gauss's divergence theorem and the no-flow boundary conditions for the mean and eddy tracer fluxes at the northern and southern lateral boundaries of the channel and the surface, it is clear that only the eddy flux across the mean isopycnal $\bar{b}=$ const remains from the mean and eddy advection in the integral balance,

$$
\int_{s_{0}}^{s_{1}} \overline{\mathbf{u}^{\prime} b^{\prime}} \cdot \mathbf{n} d s=\int_{A} d A\left(\bar{Q}-\frac{\partial}{\partial t} \bar{b}\right),
$$

where the vector $\mathbf{n}=\boldsymbol{\nabla} \bar{b}|\nabla \bar{b}|^{-1}$ points perpendicular to the mean isopycnal $\bar{b}=$ const and $s$ denotes a coordinate along the mean isopycnal from $s_{0}$ at the southern end of the channel to $s_{1}$ at the northern end of the channel. This means that if the integral on the righthand side of the integral balance vanishes; that is, if the mean diapycnal forcing $\bar{Q}$ vanishes in quasi-steady state, the integrated diapycnal eddy flux across the mean isopycnal must also vanish.

Equation (5) therefore yields an integral constraint for averaging in $z$ coordinates, similar to the previous one averaging in isopycnal coordinates. The difference is that the constraint in $z$ coordinates holds only in a (weaker) integral sense while in isopycnal coordinates the constraint holds also locally. That means that statement (i) above, which was deduced from the isopycnal framework and applied to $z$ coordinates, carries over the weaker integral constraint to a (stronger) local one in $z$ coordinates.

The way we shall implement statement (i) in this 


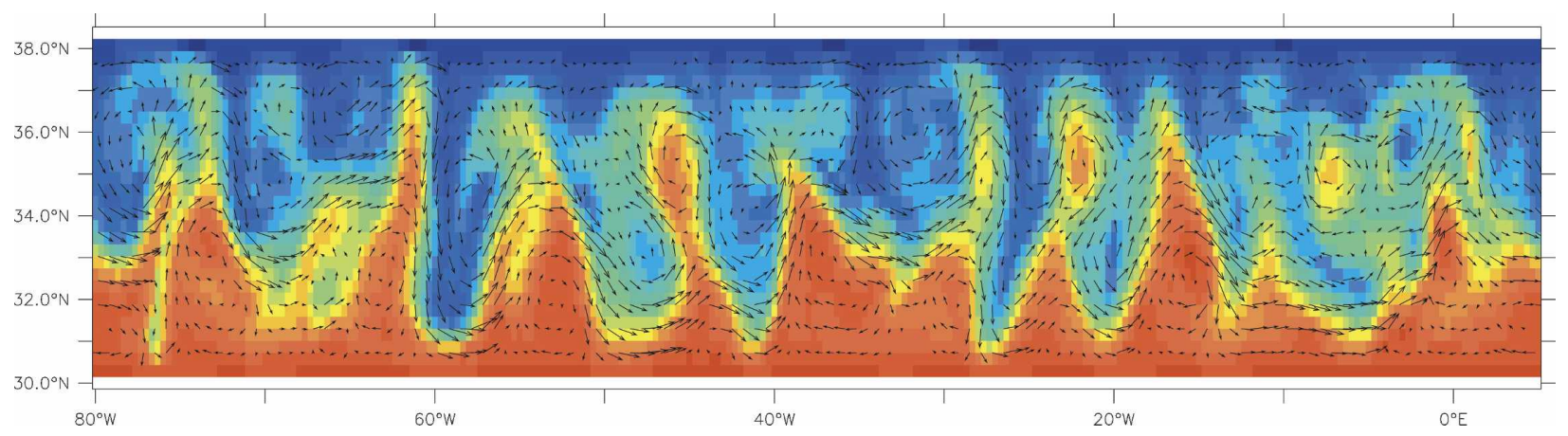

FIG. 1. Instantaneous temperature and velocity at 1000-m depth after $1 \mathrm{yr}$ of integration in experiment CHANNEL-3. Every second velocity grid point is displayed, and the color shading ranges from $2^{\circ}$ to $12^{\circ} \mathrm{C}$.

paper is by taking account of rotational fluxes in an eddy flux decomposition into advective and diffusive parts, similar to the flux decompositions of Andrews and McIntyre (1978) and McDougall and McIntosh (1996). As examples of the performance of our eddy flux interpretation we diagnose numerical experiments, which are presented in section 2 . In section 3 , we review the classical flux decomposition by Andrews and McIntyre (1978), the transformed Eulerian mean (TEM) method, and diagnose it in the numerical experiments. Section 4 presents a TEM version, which was originally proposed by Gille and Davis (1999) and is equivalent to the effective diffusivity concept by Nakamura (2001). However, while all of these TEM versions are shown to be inconsistent with statement (i), in section 5 we discuss and apply to the model a consistent and satisfactory generalization of the eddy flux interpretations of Marshall and Shutts (1981), McDougall and McIntosh (1996), and Medvedev and Greatbatch (2004). The last section concludes and discusses the results.

\section{Numerical simulations of eddy fluxes}

We are diagnosing numerical experiments using an OGCM in several idealized configurations with respect to several eddy flux decompositions. The numerical code (the code and all configurations used in this study can be found online at http://www.ifm-geomar.de/ $\sim$ spflame), which is used to integrate the OGCM, is based on a revised version of the Modular Ocean Model (MOM2; Pacanowski 1995) and formulated in $z$ coordinates.

Experiment CHANNEL-3 is a setup with a reentrant channel on a $\beta$ plane (referenced to the southernmost latitude of the model domain). Horizontal resolution is $1 / 3^{\circ}$ and there are 20 levels of $100-\mathrm{m}$ thickness. Initial conditions are a state of rest, constant meridional and vertical gradients in temperature $\left(-1 \times 10^{-5} \mathrm{~K} \mathrm{~m}^{-1}\right.$ and $8.2 \times 10^{-3} \mathrm{~K} \mathrm{~m}^{-1}$, respectively), and no zonal gradient (except for a small perturbation). A linear equation of state is used $\left(\partial \rho / \partial T=-0.2 \times 10^{-3} \mathrm{~kg} \mathrm{~m}^{-3} \mathrm{~K}^{-1}\right)$, and salinity is set to a constant. The boundary conditions are no slip at the sidewalls, and vanishing heat fluxes at the sidewalls and surface and bottom boundaries. Bottom friction following a quadratic drag law is used with a coefficient of $1.5 \times 10^{-3}$, lateral biharmonic friction is used with a viscosity of $2 \times 10^{11} \mathrm{~m}^{4} \mathrm{~s}^{-1}$, and the Quicker advection scheme (Leonard 1979) is used for the tracer with no explicit diffusion. Explicit vertical viscosity is $2 \times 10^{-4} \mathrm{~m}^{2} \mathrm{~s}^{-1}$. Temperature at the three northernmost and southernmost grid points is relaxed toward the initial condition, with a time scale ranging from 3 days at the boundary to 15 days at the outer edge of the relaxation zone.

Two further experiments are discussed in which we aim to reduce the interior diabatic forcing. Because we use no explicit diffusion in all model runs, the diabatic forcing outside the relaxation zones is due to implicit (numerical) diffusion by the advection scheme (Quicker) and other spurious numerical effects (Griffies et al. 2000; C. Eden and A. Oschlies 2006, unpublished manuscript). The most effective way to reduce these effects and correspondingly $Q$ in the model is simply given by increasing the resolution. In experiment CHANNEL-6 (CHANNEL-12) the horizontal and vertical model resolution was increased by a factor of 2 (4). In addition, the biharmonic viscosity was decreased to $1 \times 10^{11} \mathrm{~m}^{4} \mathrm{~s}^{-1}\left(2 \times 10^{10} \mathrm{~m}^{4} \mathrm{~s}^{-1}\right)$ and the temperature is relaxed toward the initial condition in 6 (12) of the southern- and northernmost grid points. All other aspects of the model are unchanged with respect to experiment CHANNEL-3.

After a couple of weeks of simulation time, baroclinic instability sets in and is producing large zonal deviations of the flow in the channel in all experiments. Figure 1 shows the fully developed stage of turbulence 
after 1-yr of integration in CHANNEL-3. In all experiments, the model is integrated for $40 \mathrm{yr}$ and temporal and zonal averages are taken for the last $30 \mathrm{yr}$ of the integration. In the following we relate $b$ to temperature, that is, density, because temperature acts as the only (active) tracer in the model.

\section{The classical eddy flux decomposition}

In this section we review the classical way of interpreting the eddy tracer flux resulting from zonal averaging at constant height. Note that all of the results carry over to temporal averaging in three dimensions. Note also that we use the following notation (Hasselmann 1982):

$$
\nabla \alpha=\left(\begin{array}{c}
\alpha_{y} \\
\alpha_{z}
\end{array}\right) \quad \text { and } \quad \nabla \alpha=\left(\begin{array}{c}
-\alpha_{z} \\
\alpha_{y}
\end{array}\right),
$$

where the subscripts $y$ and $z$ denote differentiation of the scalar $\alpha$ in the meridional and vertical direction, respectively, and the vector subscript $\neg$ denotes anticlockwise rotation by $90^{\circ}$.

The TEM method of Andrews and McIntyre (1978) introduces a decomposition of the eddy tracer flux $\mathbf{F}$ into a part aligned along contours of $\bar{b}$ and a part across contours of $\bar{b}$

$$
\overline{\mathbf{u}^{\prime} b^{\prime}} \equiv \mathbf{F}=B \nabla \bar{b} \bar{b}-K \nabla \bar{b},
$$

where $B$ and $K$ are given by projections of the eddy flux along and across contours of $\bar{b}$, respectively,

$$
\begin{aligned}
& B=|\nabla \bar{b}|^{-1} F_{\|} \quad \text { and } \\
& K=-|\nabla \bar{b}|^{-1} F_{\perp},
\end{aligned}
$$

with $F_{\perp}=\overline{\mathbf{u}^{\prime} b^{\prime}} \cdot \mathbf{n}$ the cross-isopycnal component of the eddy tracer fluxes and $F_{\|}=\overline{\mathbf{u}^{\prime} b^{\prime}} \cdot \mathbf{s}$ the alongisopycnal component of the eddy tracer fluxes, and where $\mathbf{s}=|\nabla \bar{b}|^{-1} \nabla \bar{b}$ and $\mathbf{n}=|\nabla \bar{b}|^{-1} \boldsymbol{\nabla} \bar{b}$ denote the unit vectors along and across the $\bar{b}$ contours, respectively.

The TEM eddy flux decomposition can be found in Andrews and McIntyre (1978) and is used, for instance, by Nakamura (2001), Greatbatch (2001), and Olbers and Visbeck (2005). In TEM, $B$ acts as a streamfunction for the eddy-induced tracer advection velocity $\mathbf{u}_{\text {eddy }}=$ $-\nabla B$. The sum $\mathbf{u}_{\text {eddy }}+\overline{\mathbf{u}}$ is sometimes called the "residual velocity." It is the residual velocity that advects the mean tracer $\bar{b}$, and, in addition, an eddy-induced diffusion term with diffusivity $K$ shows up in the mean tracer budget

$$
\bar{b}_{t}+(\overline{\mathbf{u}}-\nabla B) \cdot \nabla \bar{b}=\bar{Q}+\nabla \cdot K \nabla \bar{b} .
$$

The eddy-induced diffusivity $K$ vanishes only for $\mathbf{F}$. $\boldsymbol{\nabla} \bar{b}=0$, that is, if the eddy fluxes have locally no cross-isopycnal component. In the limit $\left|\bar{b}_{z}\right| \gg\left|\bar{b}_{y}\right|$, that is, for a strongly stratified situation, the eddy streamfunction becomes $B=-\overline{v^{\prime} b^{\prime}} / \bar{b}_{z}$ (Andrews and McIntyre 1976), while for $\left|\bar{b}_{y}\right| \gg\left|\bar{b}_{z}\right|$, for example, in the oceanic mixed layer, the eddy streamfunction becomes $B=\overline{w^{\prime} b^{\prime}} \bar{b}_{y}$ (Held and Schneider 1999). However, it should be stressed that the flux decompo-

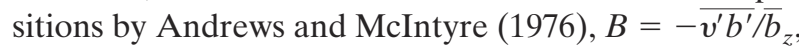
and Held and Schneider (1999), $B={\overline{w^{\prime} b^{\prime}}}_{\bar{b}} \bar{b}_{y}$, are both choices for the streamfunction $B$ in their own right and should not be viewed as approximations to the TEM.

Figure 2a shows the (residual) streamfunction for the total flow $(\overline{\mathbf{u}}-\nabla B)$ in experiment CHANNEL-3. The value for the residual streamfunction ranges from 0 to $-30 \mathrm{~m}^{2} \mathrm{~s}^{-1}$. Note that this streamfunction is given for the zonally averaged flow, and that a value of $30 \mathrm{~m}^{2} \mathrm{~s}^{-1}$ corresponds to about $30 \mathrm{~Sv}\left(\mathrm{~Sv} \equiv 10^{6} \mathrm{~m}^{3} \mathrm{~s}^{-1}\right)$ per $10^{\circ}$ longitude volume transport in this model setup. The streamfunction of the Eulerian mean flow in CHANNEL-3 $\left(-\nabla B_{m}=\overline{\mathbf{u}}\right.$, not shown explicitly) in the interior of the channel is much smaller (only up to 3 $\mathrm{m}^{2} \mathrm{~s}^{-1}$ ) than the eddy streamfunction $B$; only in the restoring zones does the mean streamfunction reach values comparable to the eddy streamfunction. Thus, the meridional overturning in the interior of the channel is dominantly eddy driven in this setup. Note that in addition to the meridional overturning, there is a strong zonal transport of about $400 \mathrm{~Sv}$ (not shown).

The residual streamfunction (Fig. 2a) shows rather strong cross-isopycnal flow in the interior of the channel. Consequently, the diffusivity $K$ in TEM is rather large; Fig. 2d shows the diagnosed eddy-induced diffusivity $(K)$ in TEM for experiment CHANNEL-3, with values of more than $100 \mathrm{~cm}^{2} \mathrm{~s}^{-1}$ at maximum in the interior of the channel. Note that $K$ inside the restoring zones is greatly exceeding $100 \mathrm{~cm}^{2} \mathrm{~s}^{-1}$ and that $K$ in TEM becomes negative in some interior regions.

Going to a higher resolution (and therefore less spurious diabatic forcing $Q$ ) changes the results only slightly. Figure $3 \mathrm{a}$ and Fig. 4a show the residual streamfunction in TEM in experiments CHANNEL-6 and CHANNEL-12, respectively, and Fig. 3d and Fig. 4d show the respective eddy-induced diffusivities $K$ in TEM. There is still strong cross-isopycnal flow in the residual streamfunctions and considerably large values of $K$ in the higher-resolution model configurations. Note that the regions in which $K$ is negative remain more or less the same in all experiments and, 
a)

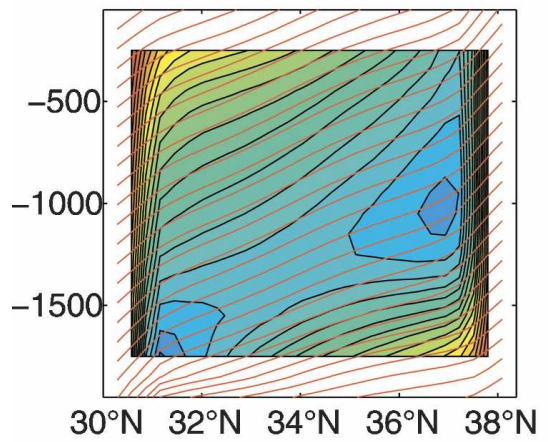

d)

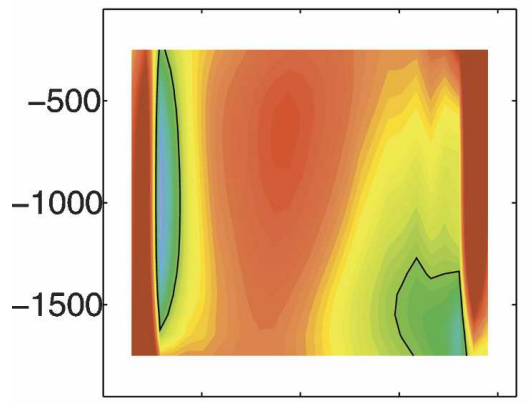

b)
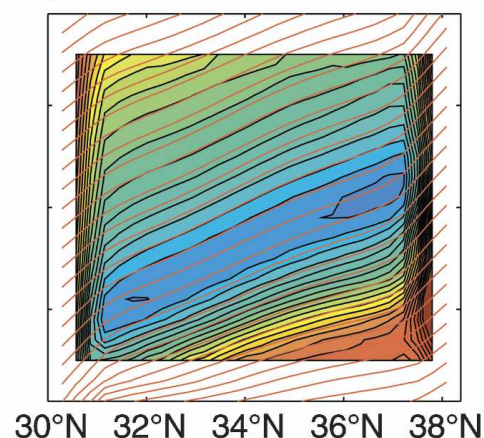

e)

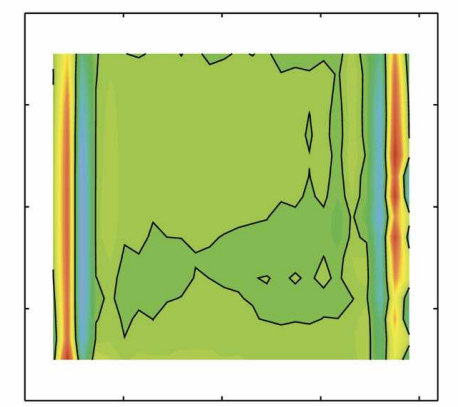

c)

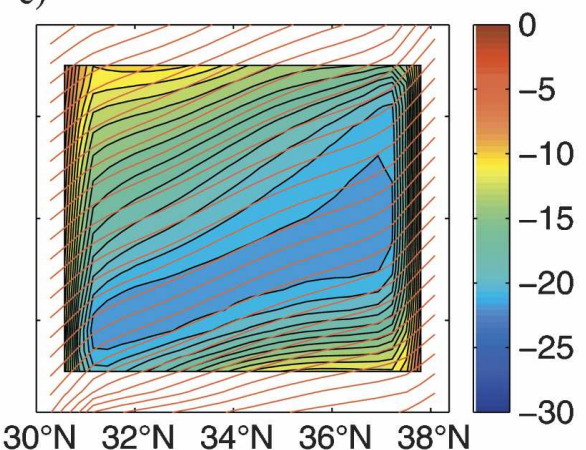

f)

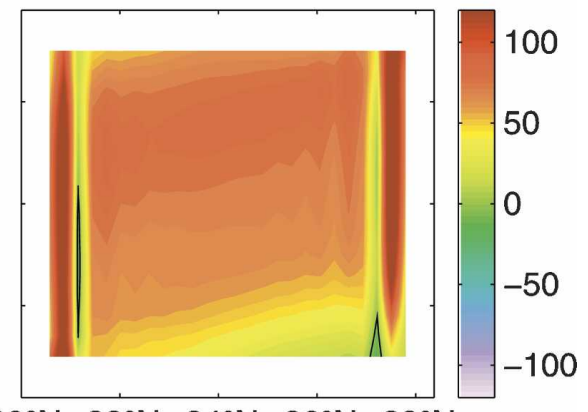

$30^{\circ} \mathrm{N} 32^{\circ} \mathrm{N} 34^{\circ} \mathrm{N} 36^{\circ} \mathrm{N} 38^{\circ} \mathrm{N} 30^{\circ} \mathrm{N} 32^{\circ} \mathrm{N} 34^{\circ} \mathrm{N} 36^{\circ} \mathrm{N} 38^{\circ} \mathrm{N} 30^{\circ} \mathrm{N} 32^{\circ} \mathrm{N} 34^{\circ} \mathrm{N} 36^{\circ} \mathrm{N} 38^{\circ} \mathrm{N}$

FIG. 2. (top) Streamfunction for the total flow in the (a) classical TEM $\left(\mathrm{m}^{2} \mathrm{~s}^{-1}\right)$, (b) TEM-A, and (c) TEM-D in experiment CHANNEL-3. Contour interval is $1 \mathrm{~m}^{2} \mathrm{~s}^{-1}$. Also shown are contours of $\bar{b}$ (red lines). (bottom) Eddy-induced diffusivity $K\left(\mathrm{~cm}^{2} \mathrm{~s}^{-1}\right.$ ) in the (d) classical TEM, (e) TEM-A, and (f) TEM-D.

if anything, show evidence of expanding into the interior.

In TEM, the eddy-induced diffusivity $K$ vanishes only if the eddy tracer fluxes are directed entirely along isopycnals. It is, however, unclear if the fluxes will locally satisfy $\boldsymbol{F} \cdot \boldsymbol{\nabla} \bar{b}=0$ in the adiabatic limit when $Q=$ 0 ; that is, it is unclear if statement (i) is satisfied by TEM. Using the TEM flux decomposition in the integral constraint [Eq. (5)],

$$
\int_{s_{0}}^{s_{1}} \overline{\mathbf{u}^{\prime} b^{\prime}} \cdot \mathbf{n} d s=-\int_{s_{0}}^{s_{1}} K|\nabla \bar{b}| d s=\int_{A} d A\left(\bar{Q}-\frac{\partial}{\partial t} \bar{b}\right)
$$

it becomes clear that only a "mean" $K$ in TEM averaged along a mean isopycnal (and weighted by $|\nabla \bar{b}|$ ) becomes zero in the adiabatic and quasi-steady case, while this cannot be shown for the local value of $K$. In our numerical experiments, which are in quasi-steady state, in TEM $K$ is large and fluctuates around zero on a mean isopycnal, and this behavior does not decrease when going to less diabatic flow regimes in higher resolutions. Thus, on the basis of our numerical experiments in which we have done our best to come close to the adiabatic case, we conclude that TEM is not in accordance with statement (i).

\section{Rotational eddy fluxes}

\section{a. Motivation}

We demonstrate in this section that large rotational eddy fluxes are the reason for the large $K$ with a fluctuating sign along a mean isopycnal in the classical TEM formalism and show the related inconsistency of TEM with statement (i). Such rotational eddy fluxes do not effect the mean tracer budget [Eq. (3)], but do effect the definitions for $B$ and $K$. Consider the flux decomposition

$$
\mathbf{F}=\underset{\neg}{\nabla} \theta+B \nabla \bar{\nabla} \bar{b}-K \boldsymbol{\nabla} \bar{b},
$$

where $\nabla \theta$ serves as a rotational gauge flux that drops out from the divergence of $\mathbf{F}$. Note that Eq. (12) is not a classical Helmholtz decomposition, because we are not insisting that all of the rotational part of the eddy tracer flux $\mathbf{F}$ is carried by $\nabla \theta$. The remainder $F-\nabla \theta$ might still show some rotational part. The eddy streamfunction $B$ and diapycnal diffusivity $K$ are now given by 
a)

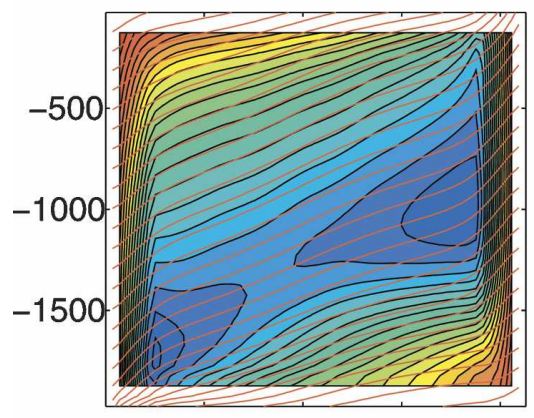

$30^{\circ} \mathrm{N} 32^{\circ} \mathrm{N} 34^{\circ} \mathrm{N} 36^{\circ} \mathrm{N} 38^{\circ} \mathrm{N} 30^{\circ}$

d)

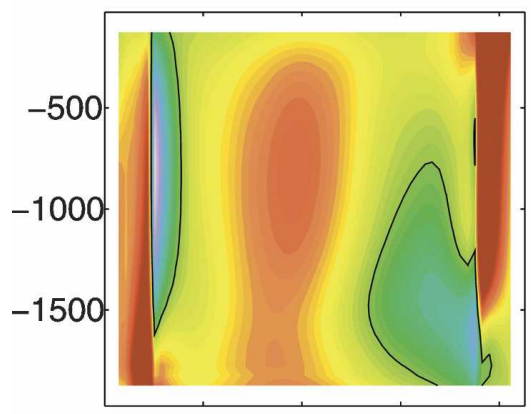

b)

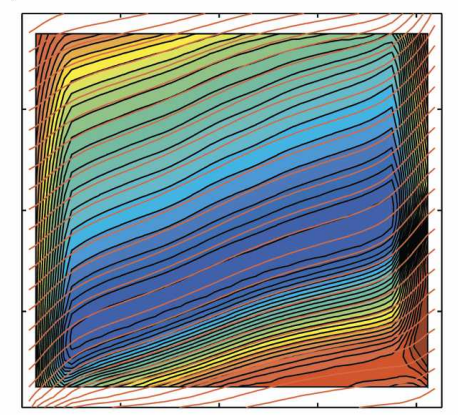

e)

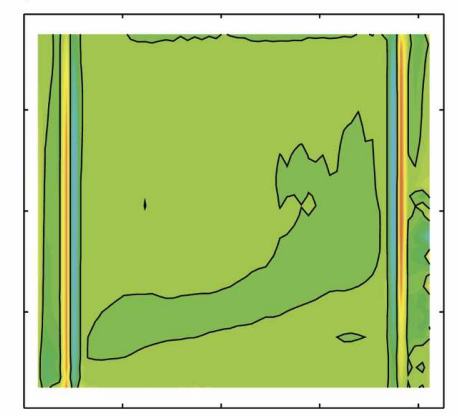

c)

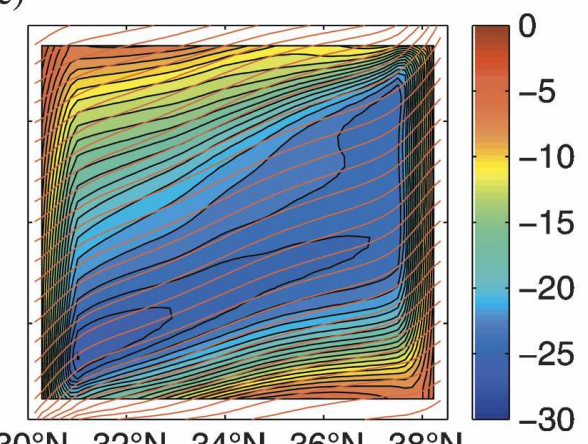

f)

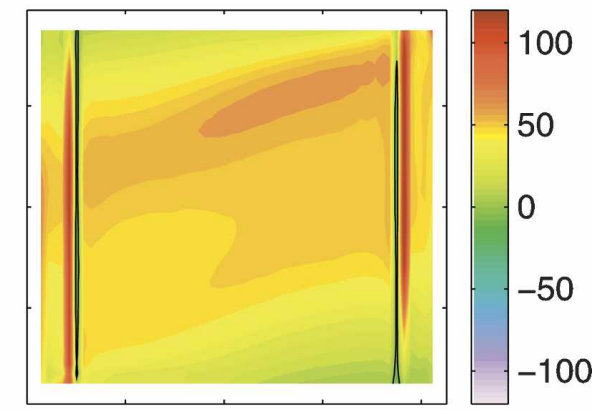

$30^{\circ} \mathrm{N} 32^{\circ} \mathrm{N} 34^{\circ} \mathrm{N} 36^{\circ} \mathrm{N} 38^{\circ} \mathrm{N} 30^{\circ} \mathrm{N} 32^{\circ} \mathrm{N} 34^{\circ} \mathrm{N} 36^{\circ} \mathrm{N} 38^{\circ} \mathrm{N} 30^{\circ} \mathrm{N} 32^{\circ} \mathrm{N} 34^{\circ} \mathrm{N} 36^{\circ} \mathrm{N} 38^{\circ} \mathrm{N}$

FIG. 3. Same as in Fig. 2, but for experiment CHANNEL-6.

$B=|\boldsymbol{\nabla} \bar{b}|^{-2}(\mathbf{F}-\underset{\sim}{\nabla} \theta) \cdot \underset{ᄀ}{\nabla} \bar{b}=|\boldsymbol{\nabla} \bar{b}|^{-1}\left(F_{\|}-\frac{\partial}{\partial n} \theta\right)$

and

$K=-|\boldsymbol{\nabla} \bar{b}|^{-2}(\mathbf{F}-\underset{\urcorner}{\nabla} \theta) \cdot \boldsymbol{\nabla} \bar{b}=-|\nabla \bar{b}|^{-1}\left(F_{\perp}+\frac{\partial}{\partial s} \theta\right)$,

introducing the along- $(\partial / \partial s)()=\mathbf{s} \cdot \boldsymbol{\nabla}()$, and cross- $(\partial /$ $\partial n)()=\boldsymbol{n} \cdot \boldsymbol{\nabla}()$, isopycnal derivatives.

We will discuss here a choice for $\theta$ that was originally proposed by Gille and Davis (1999) (their $\Psi_{\text {opt }}$ ) and corresponds to the effective eddy diffusivity concept of Nakamura (2001). This modification of the classical TEM for diabatic flow is called hereafter TEM-D. The rotational gauge potential $\theta$ in TEM-D follows from a minimization of the eddy-induced diffusivity as outlined in appendix A. The difference between this and the classical TEM is that it is not the local diapycnal eddy flux $F_{\perp}$, which determines $K$, but the averaged flux along an isopycnal, $\int_{s_{0}}^{s_{1}} F_{\perp}(s, n) d s$. As seen in the integral budget [Eq. (5)], this integrated flux goes to zero for the adiabatic and quasi-steady case.

\section{b. The adiabatic TEM}

We begin by discussing the simple choice

$$
\theta=-\int_{s_{0}}^{s} F_{\perp} d s^{\prime}
$$

for which we obviously get $K=0$ in Eq. (14). In the quasi-steady case $\bar{b}_{t}=0$, the residual flow in the mean tracer budget is then purely along contours of $\bar{b}$, and for $Q=0$, the choice would be in agreement with statement (i) from section 1 . We call this flux decomposition the adiabatic TEM, thus TEM-A.

Although in principle mathematically valid, it is shown in appendix B that when applying TEM-A to diabatic flows, that is, with $Q \neq 0$, the integrated crossisopycnal eddy flux is redirected as a rotational flux through the boundary of the channel, which is a rather unphysical result. Only in quasi-steady, purely adiabatic conditions is the integrated cross-isopycnal eddy flux zero [Eq. (5)] and TEM-A an acceptable flux decomposition. In fact, TEM-A nicely demonstrates that setting $K=0$ in quasi-steady flows is physically justified only for the (hypothetical) adiabatic case, that is, if $Q=0$.

Clearly, in reality there is always diabatic forcing present and the numerical experiments discussed here certainly contain diabatic forcing. Therefore, TEM-A does not appear to be a reasonable eddy flux interpretation for our application. However, TEM-A remains a useful diagnostic tool to deduce the mean diabatic forcing $\bar{Q}$ in the numerical experiments. Note that estimating the "real" diabatic forcing $\bar{Q}$ in numerical models is rather difficult (Griffies et al. 2000; C. Eden and A. 
a)

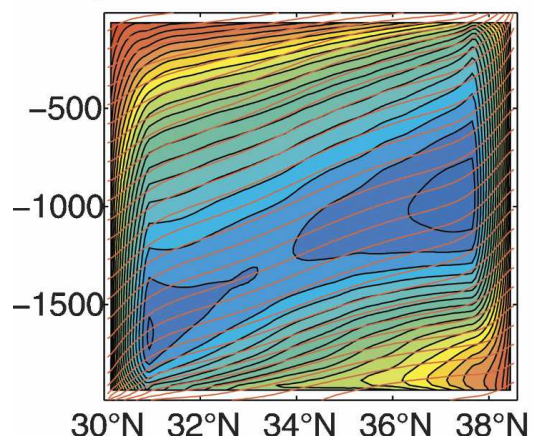

d)

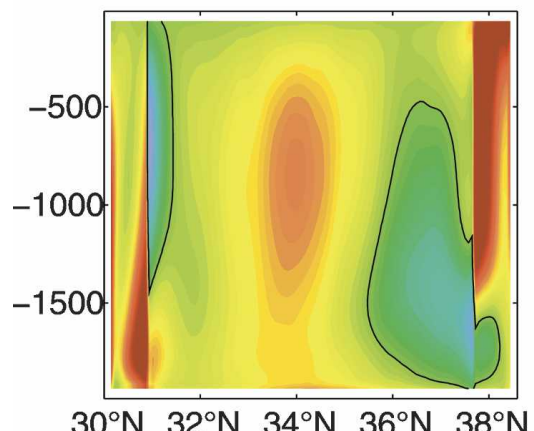

b)

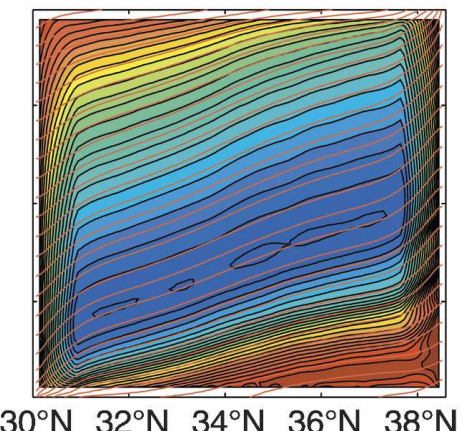

e)

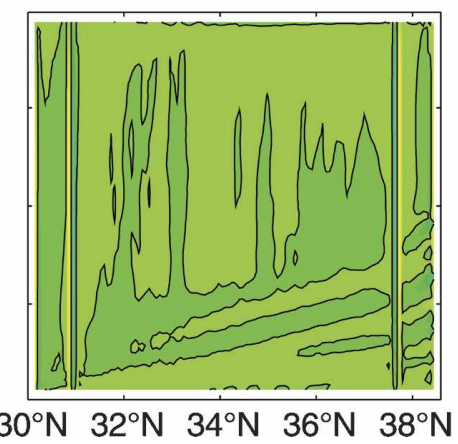

c)

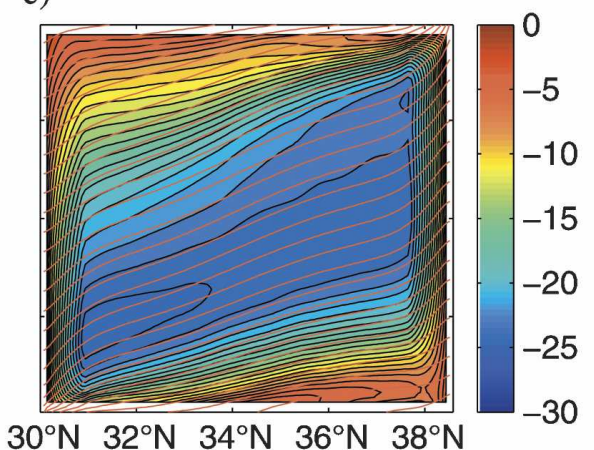

f)

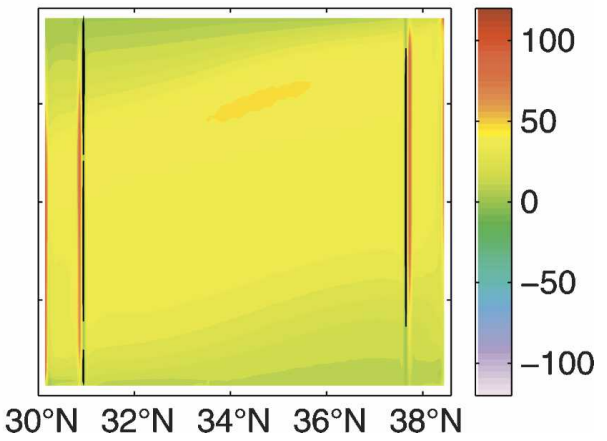

FIG. 4. Same as in Fig. 3, but for experiment CHANNEL-12.

Oschlies 2006, unpublished manuscript), because there are no numerical advection schemes (even without any implicit diffusion, such as the classical "leapfrog" scheme) that show the same properties as analytical advection, with the consequence that errors in the schemes have to be interpreted as diabatic forcing.

Applying TEM-A in the quasi-steady diabatic case yields

$$
(\overline{\mathbf{u}}-\underset{\urcorner}{\nabla} B) \cdot \nabla \bar{b}=\bar{Q},
$$

that is, only a nonzero $\bar{Q}$ can force cross-isopycnal residual flow, which is a feature of TEM-A we now exploit for diagnosing the numerical experiments. Our aim is to compare $\bar{Q}$ with the eddy-induced mixing $\boldsymbol{\nabla} \cdot K \boldsymbol{\nabla} \bar{b}$ in the classical TEM case. Figure $2 \mathrm{~b}$ shows the (residual) streamfunction for the total (meridional) flow $(\overline{\mathbf{u}}-\nabla B)$ for TEM-A in experiment CHANNEL3. In contrast to the classical TEM (Fig. 2a), the residual flow of TEM-A in the interior of the channel is more or less aligned along isopycnals with very little flow across isopycnals. Only inside the restoring zones does the residual streamfunction in TEM-A show large crossisopycnal flow, while the eddy-induced diffusivity $K$ in
TEM-A (Fig. 2e) fluctuates around zero diapycnal diffusivity, as expected by the construction. ${ }^{5}$

In TEM-A any cross-isopycnal (quasi steady) flow is entirely driven by $\bar{Q}$. Therefore, we can conclude that the term $\boldsymbol{\nabla} \cdot K \nabla \bar{b}$ in TEM drives the strong crossisopycnal residual flow in the interior of the channel evident in Figs. 2a, 3a, and 4a. Thus, $K$ in the classical TEM must be much larger than an equivalent (mean) diapycnal diffusivity implied by $\bar{Q}$. When going to higher resolutions in CHANNEL-6 and CHANNEL12 , the picture hardly changes; that is, the residual streamfunction is more or less aligned along mean isopycnals in the interior of the channel, indicating that $\bar{Q}$ does not change much when going to higher resolutions.

\section{c. The diabatic TEM}

Now we return to the TEM-D eddy flux interpretation, originally proposed by Gille and Davis (1999),

\footnotetext{
${ }^{5}$ Inside the restoring zones near the sidewalls of the channel, however, larger residuals of $K$ in TEM-A show up for numerical reasons. Note that this artifact vanishes, when going to a higher resolution (as in experiment CHANNEL-6 and CHANNEL-12, discussed below). Note that for the TEM-D (see below) the same artifact shows up in CHANNEL-3 and vanishes as well in CHANNEL-6 and CHANNEL-12.
} 
which corresponds to the effective diffusivity concept of Nakamura (2001). Figure 2c shows the (residual) streamfunction for the total (meridional) flow $(\overline{\mathbf{u}}-\nabla B)$ in TEM-D in experiment CHANNEL-3. The crossisopycnal residual flow ranges between the strong cross-isopycnal flow in the classical TEM (Fig. 2a) and the almost-vanishing cross-isopycnal flow in TEM-A (Fig. 2b). Figure $2 \mathrm{f}$ shows the diagnosed eddy-induced diapycnal diffusivity $K$ in TEM-D for experiment CHANNEL-3. It ranges in between the extreme cases of TEM and TEM-A, as expected from the cross-isopycnal flow of the residual streamfunctions. Note that while $K$ in TEM becomes negative in some regions, this is not the case for TEM-D. Because $|\nabla \bar{b}|$ does not vary much in our numerical experiments, $K$ in TEM-D shows almost no structure along a mean isopycnal. These uniform values of $K$ reduce from about $100 \mathrm{~cm}^{2} \mathrm{~s}^{-1}$ in CHANNEL-3 to about $50 \mathrm{~cm}^{2} \mathrm{~s}^{-1}$ in CHANNEL-6 and about $20 \mathrm{~cm}^{2} \mathrm{~s}^{-1}$ in CHANNEL-12. In all experiments, $K$ in TEM-D is essentially an average over the very large $K$ s of TEM inside the restoring zone near the sidewalls of the channel and the lower values in the interior.

There are strong rotational fluxes in the interior in all experiments (not shown). Note that in TEM-D the vertical rotational fluxes $[-(\partial / \partial y) \theta]$ are of the same magnitude as the advective fluxes $[-B(\partial / \partial y) \bar{b}]$ and the diffusive fluxes $[-K(\partial / \partial z) \bar{b}]$ in all experiments, and that the meridional rotational fluxes $[(\partial / \partial z) \theta]$ are of the same magnitude as the advective fluxes $[B(\partial /$ $\partial z) \bar{b}]$, while the meridional diffusive fluxes are orders of magnitude smaller. This demonstrates again the importance of the rotational fluxes in setting the shape and magnitudes of the eddy streamfunction $B$ and the diffusivity $K$. Note also that the interior rotational fluxes become stronger in magnitude when going to higher resolutions, rather than getting smaller.

TEM-D involves a nonlocal definition of the rotational gauge potential $\theta$, which leads to an eddyinduced diffusivity that contains information throughout the channel about the strong diabatic forcing in the restoring zones near the northern and southern boundaries, even in regions in the interior that might be completely adiabatic. This is somehow an unsatisfactory result, also mentioned previously by Nurser and Lee (2004), and therefore we conclude that TEM-D is also an unsatisfactory eddy flux interpretation. In the next section, we will formulate another flux decomposition, which gives a more local definition of $\theta$ and the eddyinduced diffusivity in turn.

\section{The temporal residual mean}

Adding a rotational nondivergent part to the eddy tracer flux $\mathbf{F}$ does not effect the mean tracer budget. However, it does effect the eddy variance equation, offering an opportunity to interpret this part of the flux. This fact was used by McDougall and McIntosh (1996) to develop the temporal residual mean (TRM-I) extension to the TEM theory, and later the TRM-II version (McDougall and McIntosh 2001). We discuss here the more general and, in this context, simpler derivation, similar to what can be found in Greatbatch (2001) and Medvedev and Greatbatch (2004), and compare it with the above-presented flux decompositions.

The eddy tracer fluxes are again expressed as in Eq. (12), where $\nabla \theta$ serves as a gauge flux that drops out when taking the divergence of $\mathbf{F}$ and therefore does not contribute to the mean tracer budget. Motivation for choosing a nonzero rotational potential $\theta$ comes from the budget of tracer variance $\left(\phi_{2}=b^{\prime 2} / 2\right)$, given by

$$
\left(\overline{\phi_{2}}\right)_{t}+\boldsymbol{\nabla} \cdot \overline{\mathbf{u} \phi_{2}}=\overline{b^{\prime} Q^{\prime}}-\mathbf{F} \cdot \nabla \bar{b} .
$$

Using the flux decomposition [Eq. (12)] for $\mathbf{F}$ in the variance budget yields

$$
\left(\overline{\phi_{2}}\right)_{t}+\nabla \cdot \overline{\mathbf{u} \phi_{2}}=\overline{b^{\prime} Q^{\prime}}-\nabla \theta \cdot \nabla \bar{b}+K|\nabla \bar{b}|^{2} .
$$

We now decompose the total (i.e., mean plus eddy) flux of eddy tracer variance into components along and across contours of $\bar{b}$ plus a rotational part, as we did before for the eddy tracer flux $\mathbf{F}$,

$$
\overline{\mathbf{u} \phi_{2}}=\underset{\neg}{\nabla} \theta_{2}+B_{2} \nabla \bar{b}-K_{2} \nabla \bar{b},
$$

where $B_{2}$ and $K_{2}$ are given by

$$
\begin{aligned}
& B_{2}|\nabla \bar{b}|=\overline{\mathbf{u} \phi_{2}} \cdot \mathbf{s}-\frac{\partial}{\partial n} \theta_{2} \quad \text { and } \\
& K_{2}|\nabla \bar{b}|=-\overline{\mathbf{u} \phi_{2}} \cdot \mathbf{n}-\frac{\partial}{\partial s} \theta_{2} .
\end{aligned}
$$

As before, $\mathbf{s}$ and $\mathbf{n}$ denote unit vectors along and perpendicular to the $\bar{b}$ contours, and $(\partial / \partial s)()=\mathbf{s} \cdot \boldsymbol{\nabla}()$ and $(\partial / \partial n)()=\mathbf{n} \cdot \boldsymbol{\nabla}()$ denote along- and across-isopycnal derivatives, respectively.

This yields the following set of equations:

$$
\begin{aligned}
& \bar{b}_{t}+(\overline{\mathbf{u}}-\boldsymbol{\nabla} B) \cdot \boldsymbol{\nabla} \bar{b}=\bar{Q}+\boldsymbol{\nabla} \cdot K \boldsymbol{\nabla} \bar{b} \text { and } \\
& \left(\overline{\phi_{2}}\right)_{t}+\nabla\left(\theta-B_{2}\right) \cdot \nabla \bar{b}=\overline{b^{\prime} Q^{\prime}}+K|\nabla \bar{b}|^{2}+\nabla \cdot K_{2} \nabla \bar{b} .
\end{aligned}
$$


As before, we utilize the gauge freedom, now for $\theta$ and $\theta_{2}$, to rephrase the mean tracer [Eq. (22)] and variance [Eq. (23)] budgets. It is clear from Eq. (23) that it is natural to choose

$$
\theta=B_{2},
$$

such that the rotational gauge potential is related to the along-isopycnal flux of variance by Eq. (20). Note that this choice follows the ideas of Marshall and Shutts (1981), McDougall and McIntosh (1996), and Medvedev and Greatbatch (2004). Second, we have to specify the rotational eddy variance flux given by $\nabla \theta_{2}$.

Medvedev and Greatbatch (2004), in their version of the TRM, put $\theta_{2}$ to zero [a feature also of McDougall and McIntosh (1996), to whose approach Medvedev and Greatbatch (2004) is closely related; see below]. It is shown below that setting $\theta_{2}$ to zero is inconsistent with statement (i) for similar reasons as applied to the classical TEM discussed earlier. Analogous to the discussion of TEM-A and TEM-D, it is possible to define analogous TRM versions, namely, an "adiabatic" TRM (TRM-A hereinafter) and a "diabatic" TRM (TRM-D hereinafter). However, this route is not discussed in detail here; we just note the following features of such flux decompositions.

For TRM-A, all of the cross-isopycnal flux of variance is absorbed into a rotational flux, and $K_{2}=0$, while for the TRM-D version we would get again an "isopycnally averaged" version of $K_{2}$, that is, $K_{2}=$ $-|\nabla \bar{b}|^{-1} G_{2}(n)$ with $G_{2}=\left(s_{1}-s_{0}\right)^{-1} \int_{s_{0}}^{s_{1}} \overline{\mathbf{u} \phi_{2}} \cdot \mathbf{n} d s$. For TRM-A in general, for diabatic flows, there are rotational fluxes of variance across the sidewalls, given by $G_{2}$, but which are zero in TRM-D. Again analogous to the discussion about TEM-A/D, it is possible to show that this cross-boundary rotational flux of variance will go to zero in the quasi-steady and adiabatic limit, by considering the integral budgets of eddy and mean tracer variance above a mean isopycnal. Furthermore, in the quasi-steady and adiabatic limit, TRM-A and TRM-D are identical. In fact, in this limit $K_{2}=0$ and $K$ $=0$, and all four flux decompositions (TRM-A, TRMD, TEM-A, and TEM-D) collapse into the same, identical decomposition.

On the other hand, it is also easy to see that setting $\theta_{2}=0$, as in the "TRM-M" version of Medvedev and Greatbatch (2004), $K_{2}$ will be given by the divergence of the cross-isopycnal flux of variance, which is not guaranteed to be locally zero in the quasi-steady and adiabatic limit. Thus, from the variance budget [Eq. (23)] it follows that for TRM-M, $K$ will also not necessarily be zero in the quasi-steady and adiabatic limit; that is, TRM-M is inconsistent with statement (i). It should also be noted that the difference between
TRM-M and TRM-I (McDougall and McIntosh 1996) is given by assuming that in TRM-I $\overline{\mathbf{u}^{\prime} \phi_{2}} \approx 0$.

It is possible to extend the ideas of Greatbatch (2001) to the full hierarchy of moments that yields a generalized form of the TRM along the ideas of Marshall and Shutts (1981), McDougall and McIntosh (1996), and Greatbatch (2001) and avoids any nonlocal definition of the rotational gauge potential $\theta$. This eddy flux decomposition is called the generalized temporal residual mean (TRM-G).

The hierarchy of tracer moments is given by

$$
\left(\overline{\phi_{n+1}}\right)_{t}+\boldsymbol{\nabla} \cdot \overline{\mathbf{u} \phi_{n+1}}+n \overline{\phi_{n} \mathbf{u}} \cdot \nabla \bar{b}=n \overline{\phi_{n} Q}-n \bar{\phi}_{n} \bar{b}_{t},
$$

with $\phi_{n}=b^{\prime n} / n$. Note that the advective terms and the dissipation term in Eq. (25) contain the full velocity $\mathbf{u}$ and the full forcing $Q$. As before, for the eddy tracer flux $\mathbf{F}$ [Eq. (12)] and the eddy tracer variance flux $\overline{\mathbf{u} \phi_{2}}$ [Eq. (19)], a similar flux decomposition is also applied to the fluxes of higher-order variances

$$
\overline{\mathbf{u} \phi_{n}}=\underset{\urcorner}{\nabla} \theta_{n}+B_{n} \nabla \bar{\neg} \bar{b}-K_{n} \nabla \bar{b},
$$

which yields the following set of equations:

$$
\begin{aligned}
\bar{b}_{t}+\underset{\sim}{(\overline{\mathbf{u}}-\underset{\sim}{\nabla} B) \cdot \nabla \bar{b}}=\bar{Q}+\boldsymbol{\nabla} \cdot K \boldsymbol{\nabla} \bar{b}, \\
\left(\phi_{2}\right)_{t}+\underset{\neg}{\boldsymbol{\nabla}}\left(\theta-B_{2}\right) \cdot \nabla \bar{b}=\overline{b^{\prime} Q^{\prime}}+K|\nabla \bar{b}|^{2}+\nabla \cdot K_{2} \nabla \bar{b},
\end{aligned}
$$

and

$$
\begin{aligned}
\left(\overline{\phi_{n+1}}\right)_{t}+\underset{\urcorner}{\nabla}\left(n \theta_{n}-B_{n+1}\right) \cdot \nabla \bar{b}= & n \overline{\phi_{n} Q}-n \bar{\phi}_{n} \bar{b}_{t}+ \\
& +n K_{n}|\nabla \bar{b}|^{2} \\
& +\nabla \cdot K_{n+1} \nabla \bar{b}
\end{aligned}
$$

Note that Eq. (29) gives the budget for the higher-order moments $\overline{\phi_{n+1}}$ using the flux decomposition [Eq. (26)], and the other two budgets are simply a repetition from above. We extend the ideas of Greatbatch (2001) and set $\theta=\mathrm{B}_{2}$ and $n \theta_{n}=B_{n+1}$ in the full hierarchy of equations.

Introducing the along-isopycnal fluxes $J_{n}=\overline{\mathbf{u} \phi_{n}} \cdot \mathbf{s}$, and using the setting

$$
n \theta_{n}=B_{n+1}=|\nabla \bar{b}|^{-1}\left(J_{n+1}-\frac{\partial}{\partial n} \theta_{n+1}\right)
$$

iteratively in Eqs. (13) and (14) for the eddy streamfunction $B$ and the eddy-induced diffusivity $K$, respectively, yields the following set of equations for $B, K$, and the rotational gauge potential $\theta$ in the TRM-G: 


$$
B|\nabla \bar{b}|=F_{\|}-\frac{\partial}{\partial m} J_{2}+\frac{1}{2}\left(\frac{\partial}{\partial m}\right)^{2} J_{3}-\frac{1}{3 !}\left(\frac{\partial}{\partial m}\right)^{3} J_{4}+\cdots,
$$

$K|\nabla \bar{b}|=-F_{\perp}-\frac{\partial}{\partial s} \theta, \quad$ and

$$
\theta|\nabla \bar{b}|=J_{2}-\frac{1}{2} \frac{\partial}{\partial m} J_{3}+\frac{1}{3 !}\left(\frac{\partial}{\partial m}\right)^{2} J_{4}-\cdots,
$$

introducing the operator $(\partial / \partial m)()=(\partial / \partial n)|\nabla \bar{b}|^{-1}()$. Using the variance budgets [Eq. (29)], we can express the eddy-induced diapycnal diffusivity in quasi-steady (but diabatic) conditions also as

$$
\begin{aligned}
K|\nabla \bar{b}|^{2}= & -\overline{b^{\prime} Q^{\prime}}+\mathcal{D}\left(\overline{\phi_{2} Q}\right)-\frac{1}{2} \mathcal{D}^{2}\left(\overline{\phi_{3} Q}\right)+\frac{1}{3 !} \mathcal{D}^{3}\left(\overline{\phi_{4} Q}\right) \\
& -\frac{1}{4 !} \mathcal{D}^{4}\left(\overline{\phi_{4} Q}\right)+\cdots
\end{aligned}
$$

or for the adiabatic (but unsteady) regime as

$$
\begin{aligned}
K|\nabla \bar{b}|^{2}= & \left(\overline{\phi_{2}}\right)_{t}-\frac{1}{2} \mathcal{D}\left(\overline{\phi_{3}}\right)_{t}+\frac{1}{3 !} \mathcal{D}^{2}\left(\overline{\phi_{4}}\right)_{t}-\mathcal{D}\left(\overline{\phi_{2}} \overline{b_{t}}\right) \\
& +\frac{1}{2} \mathcal{D}^{2}\left(\overline{\phi_{3}} \overline{b_{t}}\right)-\frac{1}{3 !} \mathcal{D}^{3}\left(\overline{\phi_{4}} \overline{b_{t}}\right)+\cdots,
\end{aligned}
$$

introducing the operator $\mathcal{D}()=\boldsymbol{\nabla} \cdot \boldsymbol{\nabla} \bar{b}|\boldsymbol{\nabla} \bar{b}|^{-2}()$. Note that for the general case (unsteady and diabatic) Eqs. (34) and (35) will simply add. TRM-G for the threedimensional case and temporal averaging is outlined in appendix C.

We see that $K$ in TRM-G will be zero in the adiabatic and quasi-steady regime, and thus TRM-G is in accordance with statement (i). As a result, we have constructed a fully consistent flux decomposition based on the ideas of Marshall and Shutts (1981), McDougall and McIntosh (1996), and Greatbatch (2001), which can be evaluated for any stratification and to any order, and gives a locally defined eddy-induced diffusivity and eddy streamfunction. This is the main result of this paper.

Note that TEM-A, TEM-D, and TRM-G collapse into a single flux decomposition in the adiabatic case. It is also easy to see that the definition for the streamfunction $B$ in TRM-G is identical to the classical TEM in the first term of the series in Eq. (31), and to TRM-M by Medvedev and Greatbatch (2004) in the first and second term. The same holds for $K$ in Eq. (32) after substituting for $\theta$ from Eq. (32).

Figure 5 shows the eddy-induced diffusivity $K$ in TRM-G, calculated in CHANNEL-6 with increasing orders in perturbation amplitude. For Fig. 5 a $K$ is evaluated to the first term in the series, that is, $K|\nabla \bar{b}|=$ $-F_{\perp}$, which is the same as $K$ in TEM; Fig. 5 b uses $K$ to the second term, which is the same as $K$ in TRM-M; likewise, Fig. $5 \mathrm{c}$ is evaluated to the third term and Fig. $5 \mathrm{~d}$ to the fourth term. Note that TRM-I of McDougall and McIntosh (1996) (not shown) is very similar to the classical TEM in our setup. There is a large decrease in magnitude of $K$ in the interior of the channel going from the first term (Fig. 5a, equivalent to TEM) to the second term (Fig. 5b, equivalent to TRM-M). However, there are still regions of negative $K$ in TRM-M. These regions are further reduced and are subsequently vanish, including higher-order terms in TRM-G also. On the other hand, the corrections to $K$ are decreasing in higher orders, showing the rapid convergence of TRM-G in our experimental setup.

Note that the diffusivity $K$ evaluated to the second term (Fig. 5b) appears to be a reasonable approximation to the full form (excluding the regions of negative $K)$. Because this $K$ is almost identical to that given by the first term in (33), that is, $K=-(\nabla \bar{b})^{-2} \bar{Q}^{\prime} b^{\prime}$, we conclude that the first term in (33) provides a reasonable expression for a local definition of $K$ to be used in a parameterization (at least in our experiments). Note also that there are still very large values of $K$ in TRM-G inside the restoring zones, much larger than the "mean" value of $K$ in TEM-D. On the other hand, interior values of $K$ are small in TRM-G in the range of about 20 $\mathrm{cm}^{2} \mathrm{~s}^{-1}$, that is, much smaller than the corresponding averaged values of $K$ in TEM-D. Figure 6 shows the residual streamfunction in TRM-G, calculated in CHANNEL-6 again with increasing orders in perturbation amplitude.

\section{Summary and discussion}

It was our aim, formulated in statement (i) in section 1 , to represent the effect of eddy fluxes locally as a purely isopycnal flux in the adiabatic and quasi-steady case. It was shown that this is not guaranteed to be the case for the classical TEM formalism by Andrews and McIntyre (1978) and, as shown in numerical experiments, the implied eddy-induced diapycnal diffusion can become large with large changes of sign on a mean isopycnal. The experiments also show that this behavior does not reduce when going to higher resolutions, and thus less diabatic forcing. We have identified rotational eddy tracer fluxes as being responsible for this behavior.

Setting all diffusive eddy effects in the mean tracer budget to zero by introducing a nonlocally defined rotational flux in the eddy tracer fluxes yields for the 
a)

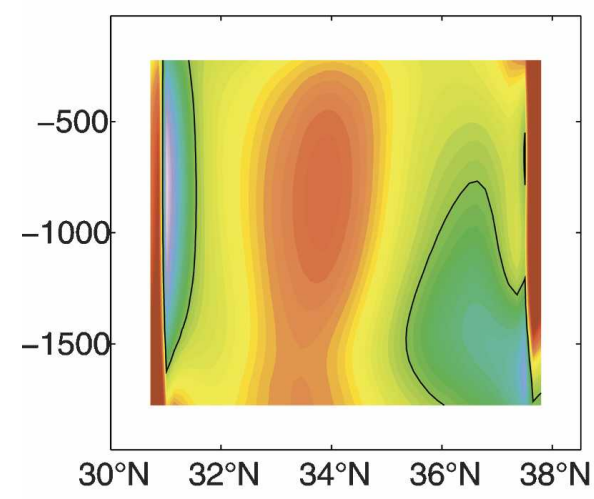

c)

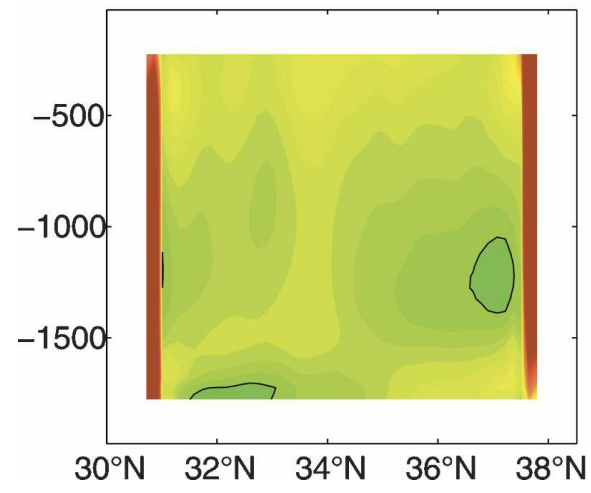

b)

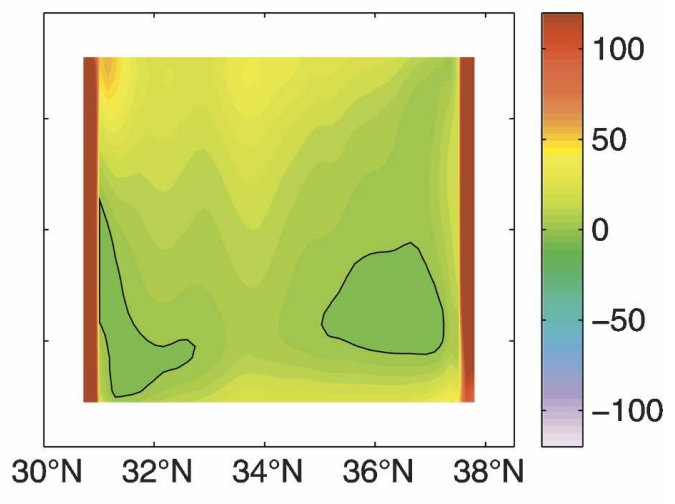

d)

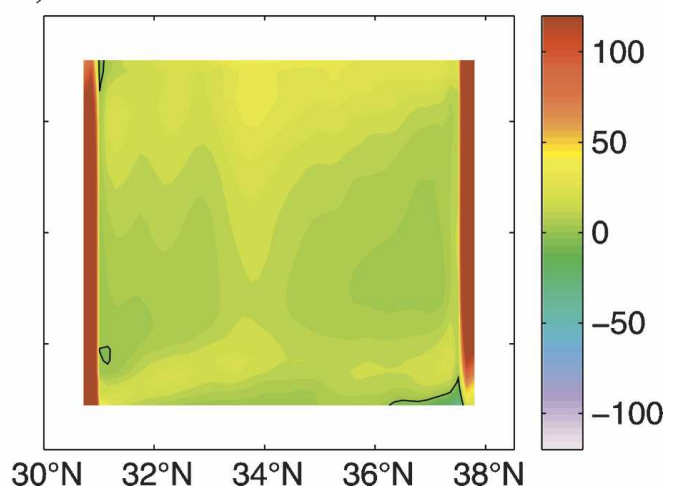

FIG. 5. Eddy-induced diffusivity $K\left(\mathrm{~cm}^{2} \mathrm{~s}^{-1}\right)$ in TRM-G calculated to (a) first, (b) second, (c) third, and (d) fourth order. Note that (a) is the same as $K$ in TEM-G and (b) is the same as $K$ in TEM-M.

(hypothetical) case of adiabatic flow only a reasonable eddy flux interpretation (thus called here TEM-A). Applying TEM-A to diabatic flows yields an unphysical cross-boundary rotational eddy tracer flux, demonstrating that implying zero eddy-induced diffusivity is physically unreasonable for diabatic flow regimes. On the other hand, the TEM-A proves to be useful as a diagnostic of the mean diabatic forcing in the numerical experiments, which is otherwise hard to estimate.

A consistent extension of the TEM-A to diabatic regimes is called TEM-D, which was originally proposed by Gille and Davis (1999) and corresponds to the effective diffusivity concept of Nakamura (2001). Here, minimal eddy-induced diapycnal diffusion shows up again in the mean tracer budget, which vanishes only in the adiabatic regime. This result points again toward the physical relevance of eddy-induced diapycnal mixing for diabatic flows. TEM-D collapses to TEM-A in the adiabatic, quasi-steady limit.

However, because the eddy-induced diffusivity in TEM-D is given by an average of the eddy tracer flux across a mean isopycnal, it can include information from strong nonlocal diabatic forcing, even in com- pletely adiabatic regions. In our numerical channel experiments, the strong forcing inside the restoring zones gives rise to large diffusivities in the interior (note that they are positive definite in all experiments). We conclude that this feature of TEM-D [and the effective diffusivity by Nakamura (2001)] is a rather unsatisfactory result. Furthermore, such a nonlocal definition of the diffusivity and streamfunction might be difficult to parameterize.

The TEM versions consider first-order moments. In contrast, the TRM originally proposed by McDougall and McIntosh (1996) considers the eddy tracer variance equation to find gauge fluxes for the eddy tracer flux. We have generalized the concept and found that TRMG, which collapses to TEM-A/D in the adiabatic limit, avoids any integral definitions of the rotational gauge fluxes. In contrast, the eddy streamfunction and the eddy-induced diffusivity in TRM-G are given by an infinite series involving fluxes of eddy tracer moments, because the flux decomposition was derived from an infinite hierarchy of budgets for tracer moments. Truncating the infinite series for the eddy-induced streamfunction and diffusivity in TRM-G after the first term 
a)

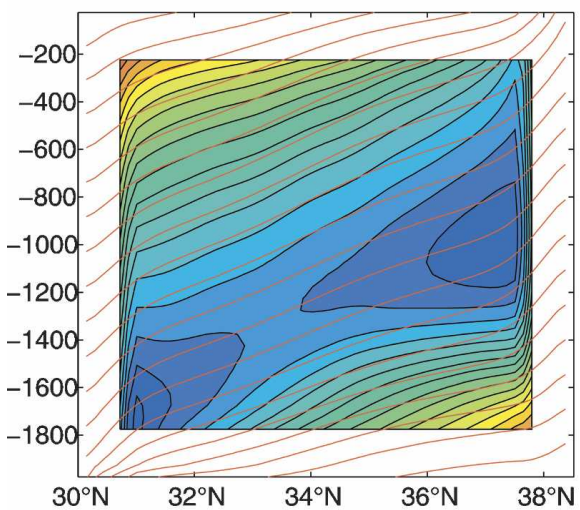

c)

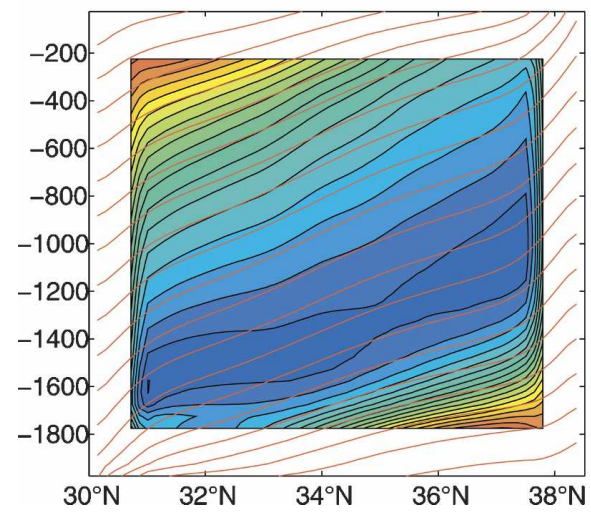

b)

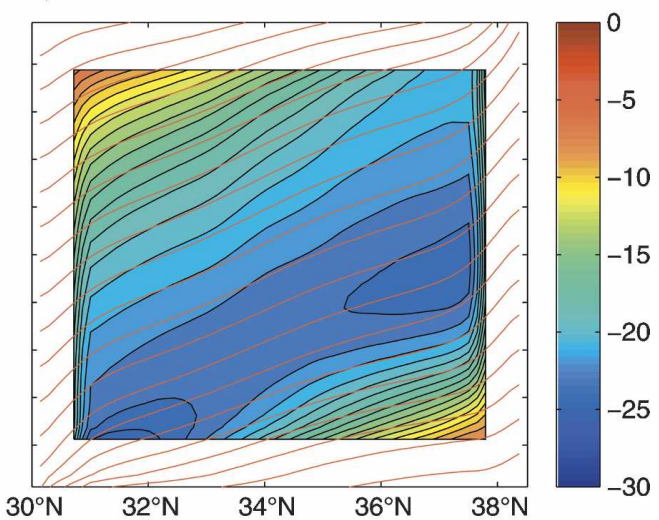

d)

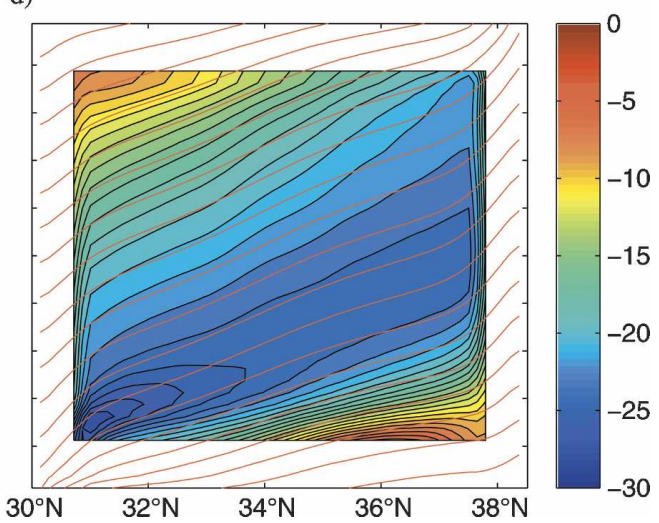

FIG. 6. Residual streamfunction $\left(\mathrm{m}^{2} \mathrm{~s}^{-1}\right)$ in TRM-G calculated to (a) first, (b) second, (c) third, and (d) fourth order. Contour interval is $1 \mathrm{~m}^{2} \mathrm{~s}^{-1}$.

gives the classical TEM, and truncating after the second term gives TRM-M by Medvedev and Greatbatch (2004) for which the special case $\left|b_{z}\right| \gg\left|b_{y}\right|$ and $\overline{v^{\prime} \phi_{2}} \approx$ 0 leads to TRM-I by McDougall and McIntosh (1996).

While TRM-M and TRM-I are not necessarily satisfying statement (i), for similar reasons as before for the classical TEM version, the new concept TRM-G is in agreement with statement (i) and can be evaluated for any stratification and to any order. It should also be noted that all arguments here carry over in a straightforward manner to three dimensions and temporal averaging, and can also be applied to isopycnal averaging to take account of rotational fluxes, for example, on an isopycnal surface.

We have formulated in statement (i) that there is no (local) diapycnal mixing by eddies unless there is some other (local) small-scale diapycnal forcing or mixing process at work. However, if there is such a small-scale diapycnal mixing, TRM-G quantifies to what extent mesoscale eddy activity is able to locally enhance (or reduce) this mixing, that is, how the large-scale mean quantities are affected by the small-scale mixing pro- cesses. TRM-G relates eddy-induced diapycnal diffusivity locally to

- either the growth or decay of mesoscale density variance and higher-order moments, and/or

- covariances between the small-scale forcing or mixing and density fluctuations.

The eddy-induced mixing effect is rather strong in our numerical model and might be overestimated, but we think that the traditional view that eddies are mixing completely adiabatically has to be revised, as proposed by Tandon and Garrett (1996) and recently by Radko and Marshall (2004). We note too that our formula for the eddy-induced diffusivity [Eq. (33)] suggests that the diffusivity can be expected to be large where the diapycnal forcing $Q$ and its associated fluctuations $Q^{\prime}$ are large, suggesting that the diffusivity is large in the surface mixed layer, but relatively small in the much more adiabatic ocean interior, an issue we explore elsewhere.

Because it is possible to extend the TRM-G theory to any tracer, the formalism thus relates, for the general case, a three-dimensional turbulent diffusivity to local 
changes and/or dissipation of tracer variance. Note that such a local relation in the tracer variance budget is also assumed and extensively used in homogeneous turbulence theory and several turbulence closures. Using TRM-G, it is possible to "localize" the variance budget also for the spatially inhomogeneous case.

Acknowledgments. We thank J. Willebrand, A. Oschlies, A. Medvedev, and S. Danilov for stimulating discussions. We also thank George Nurser, an anonymous reviewer, and the editor for helpful comments. The model integrations have been performed on a NEC-SX5 at the computing center at the University Kiel, Germany. RJG also thanks NSERC and CFCAS for financial support through an NSERC Research Grant and the Canadian CLIVAR Research Network.

\section{APPENDIX A}

\section{Minimal Cross-Isopycnal Eddy Fluxes}

Because $\boldsymbol{\nabla} \theta$ makes no contribution to the mean tracer budget, there is flexibility in the choice of $\theta$, a flexibility that is exploited here. We ask for a $\theta$ in the flux decomposition in Eq. (12) that minimizes (in some sense) $K \nabla \bar{b}$, which denotes the cross-isopycnal eddy flux, when specifying $b$ as potential density. This leads to the following minimization problem:

$$
\frac{1}{2} \int W(K \nabla \bar{b})^{2} d y d z=\min ,
$$

where $W(y, z)$ denotes a weighting function that is specified below. The corresponding Euler-Lagrange equation $^{\mathrm{A} 1}$ is

$$
\underset{\neg}{\nabla} \bar{b} \cdot \boldsymbol{\nabla}\left[\frac{W}{|\nabla \bar{b}|^{2}}(\mathbf{F} \cdot \boldsymbol{\nabla} \bar{b}+\boldsymbol{\nabla} \theta \cdot \underset{\neg}{\nabla} \bar{b})\right]=0,
$$

which states that the term inside the brackets is constant along contours of $\bar{b}$. It is convenient to use again coordinates along $(s)$ and perpendicular $(n)$ to contours of $\bar{b}$ together with the along- $(\partial / \partial s)()=\mathbf{s} \cdot \boldsymbol{\nabla}()$ and cross$(\partial / \partial n)()=\mathbf{n} \cdot \boldsymbol{\nabla}()$, isopycnal derivatives, so that the bracketed term in Eq. (A2) becomes

$$
F_{\perp}(s, n)+\frac{\partial \theta(s, n)}{\partial s}=G(n)
$$

by specifying $W$ as $W=|\nabla \bar{b}|$. We get as the optimal vector gauge potential

\footnotetext{
${ }^{\text {A1 }}$ Note that all results presented here carry over to three dimensions, including the solution of the Euler-Lagrange equation.
}

$$
\theta(s, n)=\int_{s_{0}}^{s}\left[G(n)-F_{\perp}\right] d s^{\prime}+H(n),
$$

and for the eddy-induced diapycnal diffusivity, in turn,

$$
K=-|\nabla \bar{b}|^{-1}\left(F_{\perp}+\frac{\partial \theta}{\partial s}\right)=-|\nabla \bar{b}|^{-1} G(n),
$$

with arbitrary functions $G(n)$ and $H(n)$.

Setting the integration constant $G(n)$ in Eq. (A5) to zero, we get zero eddy-induced diffusivity $K$ as the minimal value and the TEM-A version (see above and appendix B). To obtain no rotational eddy tracer flux through the boundaries in the diabatic case, we must ensure that $\theta=$ const along the boundaries, that is,

$$
G(n)=\left(s_{1}-s_{0}\right)^{-1} \int_{s_{0}}^{s_{1}} F_{\perp}(s, n) d s=F_{\mathrm{ave}}(n) .
$$

For this choice there is an eddy-induced diffusivity given by the cross-isopycnal eddy fluxes averaged along an isopycnal. The difference to the classical TEM is that it is not the local cross-isopycnal eddy flux $F_{\perp}$ that determines $K$, but the averaged flux $\int_{s_{0}}^{s_{1}} F_{\perp}(s, n) d s$. What is left in $K$ is the minimal diapycnal flux that is needed to constrain the no-flow boundary condition for the rotational eddy flux. We call this flux decomposition TEMD. Note that TEM-D was originally proposed by Gille and Davis (1999) and corresponds to the effective (eddy induced) diffusivity concept by Nakamura (2001), recently used, for instance, by Marshall et al. (2006). To diagnose TEM-D in the numerical experiments, we simply use $\theta=0$ as the boundary condition to solve Eq. (A2) using the same procedure as that for TEM-A.

TEM-D approaches the classical TEM in the following limit. If the eddy tracer flux is entirely along contours of $\bar{b}$, that is, if $\mathbf{F} \cdot \boldsymbol{\nabla} \bar{b}=0$ everywhere, the rotational potential $\theta$, and in consequence the rotational eddy tracer flux, is zero. For this case the eddy streamfunction and diffusivity (which is also zero) become the same as that in TEM. Approaching the adiabatic limit $Q=0$, TEM-D becomes TEM-A under general circumstances. Note that the classical TEM does not necessarily approach TEM-A in this limit.

\section{APPENDIX B}

\section{Rotational Eddy Fluxes across Boundaries}

Although mathematically valid in principle, applying TEM-A to diabatic flows, that is, with $Q \neq 0$, yields an inconsistency. This can be seen considering again the 
integral constraint [Eq. (5)] for diabatic flows, using the flux decomposition [Eq. (12)] with $\theta$ from Eq. (A4) with $G=0$,

$$
\left.\theta\right|_{s_{1}}-\left.\theta\right|_{s_{0}}=\int_{s_{0}}^{s_{1}} F_{\perp} d s=\int_{A} d A\left(\bar{Q}-\bar{b}_{t}\right) .
$$

This shows that starting the integration of $\theta$ at $s=s_{0}$, where the mean isopycnal intersects a lateral boundary of the channel, must yield a different value of $\theta$ at the other boundary at $s=s_{1}$, because the cross-isopycnal eddy flux does not integrate to zero for $Q \neq 0$ (and $\bar{b}_{t} \neq 0$ ). Because $\theta$ acts as a streamfunction for a rotational eddy flux, there must be a rotational eddy flux through the lateral boundary at $s_{1}$ given by $\int_{A} d A(\bar{Q}-$ $\left.\bar{b}_{t}\right)$. Thus, setting $K=0$ by the choice of $\theta$ in TEM-A for diabatic flows redirects the integrated cross-isopycnal eddy flux as a rotational flux through the boundary, which is a rather unphysical result.

To diagnose TEM-A in the numerical experiments, $\theta$ is calculated by interpolating $\mathbf{F}[y, \bar{b}(y, z)] \cdot \boldsymbol{\nabla} \bar{b} /|\boldsymbol{\nabla} \bar{b}|$ to an equidistant grid in the new coordinate $\bar{b}$ and integrating this quantity along lines of constant $\bar{b}$, starting at the southern end of the channel, where we put $\theta=0$. In this way, we obtain $\theta(y, \bar{b})=-\int_{s_{0}}^{s} F_{\perp} d s^{\prime}$ and then interpolate $\theta$ back to $z$ as a vertical coordinate. This $\theta$ serves then as the boundary condition to solve the Euler-Lagrange equation [Eq. (A2)]. Note that the last step is formally not needed, but that it smoothes the solution for $\theta$.

\section{APPENDIX C}

\section{The Three-Dimensional TRM-G}

The extension of the above discussion of TRM-G to the three-dimensional case and temporal averaging is straightforward. Its consequences are briefly outlined here. In the mean tracer budget [Eq. (3)] and the hierarchy of tracer moments [Eq. (25)], the twodimensional operator $\boldsymbol{\nabla}$ is replaced by its threedimensional equivalent $\boldsymbol{\nabla}_{3}$. The decomposition of the (three-dimensional) eddy tracer flux $\mathbf{F}$ [Eq. (12)], the eddy tracer variance flux $\overline{\mathbf{u} \phi_{2}}$ [Eq. (19)], and the fluxes of higher-order variances [Eq. (26)] becomes

$$
\overline{\mathbf{u} \phi_{n}}=\boldsymbol{\nabla}_{3} \times \boldsymbol{\theta}_{n}+\mathbf{B}_{n} \times \boldsymbol{\nabla}_{3} \bar{b}-K_{n} \boldsymbol{\nabla}_{3} \bar{b},
$$

where $\mathbf{B}_{n}$ and $\boldsymbol{\theta}_{n}$ denote three-dimensional vectors. Note that $\mathbf{B}_{1}$ becomes a vector streamfunction for the three-dimensional eddy-driven flow $\mathbf{u}^{*}=\nabla_{3} \times \mathbf{B}$, while the eddy-induced diffusivity $K_{1}$ remains to be a scalar.

The along-isopycnal fluxes of tracer moments become in three dimensions $\mathbf{J}_{n}=\mathbf{n}_{3} \times \overline{\mathbf{u} \phi_{n}}$, with $\mathbf{n}_{3}=$ $\left|\nabla_{3} \bar{b}\right|^{-1} \nabla_{3} \bar{b}$. The setting of Eq. (30) becomes

$$
n \boldsymbol{\theta}_{n}=\mathbf{B}_{n+1}=\left|\boldsymbol{\nabla}_{3} \bar{b}\right|^{-1}\left(\mathbf{J}_{n+1}-\frac{\partial}{\partial n_{3}} \theta_{n+1}\right),
$$

with $\left(\partial / \partial n_{3}\right)()=-\mathbf{n}_{3} \times\left[\nabla_{3} \times()\right]$ and the expression for the eddy streamfunction $\mathbf{B}$, eddy-induced diffusivity $K$, and vector gauge potential $\boldsymbol{\theta}$ :

$$
\begin{aligned}
\mathbf{B}\left|\boldsymbol{\nabla}_{3} \bar{b}\right|= & \mathbf{J}_{1}-\frac{\partial}{\partial m_{3}} \mathbf{J}_{2}+\frac{1}{2}\left(\frac{\partial}{\partial m_{3}}\right)^{2} \mathbf{J}_{3}-\frac{1}{3 !}\left(\frac{\partial}{\partial m_{3}}\right)^{3} \mathbf{J}_{4} \\
& +\cdots, \\
K\left|\nabla_{3} \bar{b}\right|= & -\mathbf{F} \cdot \mathbf{n}_{3}-\frac{\partial}{\partial s_{3}} \boldsymbol{\theta}, \text { and } \\
\boldsymbol{\theta}\left|\boldsymbol{\nabla}_{3} \bar{b}\right|= & \mathbf{J}_{2}-\frac{1}{2} \frac{\partial}{\partial m_{3}} \mathbf{J}_{3}+\frac{1}{3 !}\left(\frac{\partial}{\partial m_{3}}\right)^{2} \mathbf{J}_{4}-\cdots,
\end{aligned}
$$

with $\left(\partial / \partial m_{3}\right)()=\left(\partial / \partial n_{3}\right)\left|\nabla_{3} \bar{b}\right|^{-1}()$ and $\left(\partial / \partial s_{3}\right)()=$ $\mathbf{n}_{3} \cdot \boldsymbol{\nabla}_{3} \times()$.

\section{REFERENCES}

Andrews, D. G., and M. E. McIntyre, 1976: Planetary waves in horizontal and vertical shear: The generalized Eliassen-Palm relation and the zonal mean acceleration. J. Atmos. Sci., 33, 2031-2048.

_ , and —_, 1978: Generalized Eliassen-Palm and CharneyDrazin theorems for waves on axisymmetric mean flows in compressible atmosphere. J. Atmos. Sci., 35, 175-185.

Gille, S. T., and E. E. Davis, 1999: The influence of mesoscale eddies on coarsely resolved density: An examination of subgrid-scale parameterization. J. Phys. Oceanogr., 29, 11091123.

Greatbatch, R. J., 2001: A framework for mesoscale eddy parameterization based on density-weighted averaging at fixed height. J. Phys. Oceanogr., 31, 2797-2806.

Griffies, S. M., R. C. Pacanowski, and B. R. Hallberg, 2000: Spurious diapycnal mixing associated with advection in a $\mathrm{z}-$ coordinate ocean model. Mon. Wea. Rev., 128, 538-564.

Hasselmann, K., 1982: An ocean model for climate variability studies. Progress in Oceanography, Vol. 11, Pergamon, 69-92.

Held, I. M., and T. Schneider, 1999: The surface branch of the zonally averaged mass transport circulation in the troposphere. J. Atmos. Sci., 56, 1688-1697.

Leonard, B. P., 1979: A stable and accurate convective modelling procedure based on quadratic upstream interpolation. Comput. Methods Appl. Mech. Eng., 19, 59-98.

Marshall, J., and G. Shutts, 1981: A note on rotational and divergent eddy fluxes. J. Phys. Oceanogr., 11, 1677-1679.

_- E. Shuckburgh, H. Jones, and C. Hill, 2006: Estimates and implications of surface eddy diffusivity in the Southern Ocean derived from tracer transport. J. Phys. Oceanogr., 36, 1806-1821.

McDougall, T. J., 1987: Neutral surfaces. J. Phys. Oceanogr., 17, 1950-1964.

, and P. C. McIntosh, 1996: The temporal-residual-mean velocity. Part I: Derivation and the scalar conservation equation. J. Phys. Oceanogr., 26, 2653-2665.

— , and — 2001: The temporal-residual-mean velocity. Part 
II: Isopycnal interpretation and the tracer and momentum equations. J. Phys. Oceanogr., 31, 1222-1246.

Medvedev, A. S., and R. J. Greatbatch, 2004: On advection and diffusion in the mesosphere and lower thermosphere: The role of rotational fluxes. J. Geophys. Res., 109, D07104, doi:10.1029/2003JD003931.

Nakamura, N., 2001: A new look at eddy diffusivity as a mixing diagnostic. J. Atmos. Sci., 58, 3695-3701.

Nurser, A. J. G., and M.-M. Lee, 2004: Isopycnal averaging at constant height. Part I: The formulation and a case study. $J$. Phys. Oceanogr., 34, 2721-2739.

Olbers, D., and M. Visbeck, 2005: A zonally averaged model of the meridional overturning in the Southern Ocean. J. Phys. Oceanogr., 35, 1190-1205.
Pacanowski, R. C., 1995: MOM 2 documentation, user's guide and reference manual. GFDL Ocean Group Tech. Rep. 3, 232 pp.

Radko, T., and J. Marshall, 2004: Eddy-induced diapycnal fluxes and their role in the maintenance of the thermocline. J. Phys. Oceanogr., 34, 372-383.

Tandon, A., and C. Garrett, 1996: On a recent parameterization of mesoscale eddies. J. Phys. Oceanogr., 26, 406-416.

Wüst, G., 1935: Schichtung und Zirkulation des Atlantischen Ozeans: (Part 2) Die Stratosphäre. Wissenschaftliche Ergebnisse der Deutschen Atlantischen Expedition auf dem Forschungs- und Vermessungsschiff “Meteor" 1925-1927, Vol. 6, Part I, 180 pp. [Reprinted in English as W. J. Emery, Ed., 1978: The Stratosphere of the Atlantic Ocean, Amerind, 112 pp.] 